\title{
Bacteremia por Aeromonas caviae en paciente con hepatitis autoinmune: presentación de caso
}

\author{
Jorge Mario Palmezano Díaz* \\ Claudia Lucía Figueroa-Pineda ${ }^{* *}$ \\ Diego Alejandro Rangel Rivera***
}

\begin{abstract}
*Residente de III año en Medicina Interna. Hospital Universitario de Santander. Universidad Industrial de Santander. Bucaramanga. Santander. Colombia.

**Médico Internista. Maestría en epidemiología. Servicio de Medicina Interna. Hospital Universitario de Santander. Universidad Industrial de Santander. Bucaramanga. Santander. Colombia.

***Médico y cirujano. Grupo de investigación en genética humana. Universidad Industrial de Santander. Bucaramanga. Santander. Colombia.

Correspondencia: Dr. Jorge Palmezano. Dirección: Transversal 93 \# 34-180, Torre 3, Apartamento 11-01, Torres de Monterrey. Bucaramanga. Santander. Colombia. Teléfono: +57 3013382047. Correo electrónico: dar_2333@hotmail.com; palmezano96@hotmail.com
\end{abstract}

\section{RESUMEN}

La hepatitis autoinmune es una enfermedad inflamatoria hepática mediada por un mecanismo de autoinmunidad. Este trastorno tiene un amplio espectro clínico y debe ser sospechado en presencia de elevación de inmunoglobulinas y aminotransferasas en un paciente con histopatología positiva para hepatitis. El diagnóstico se realiza descartando otras causas de hepatitis (viral, secundaria a medicamentos) y complementado con los estudios de autoanticuerpos típicos de esta enfermedad. El tratamiento se fundamenta en la monoterapia con corticoesteroides o su asociación con otros inmunomoduladores, obteniéndose buenas tasas de respuesta terapéutica.

La tasa de infección hospitalaria en pacientes con cirrosis hepática oscila entre 32-34\% siendo la bacteremia la cuarta fuente más frecuente, la cual tiene como etiología al género Aeromonas en un $5 \%$. Se presenta el caso de una paciente de 51 años con hepatitis autoinmune quien consulta por descompensación de enfermedad hepática secundaria a bacteremia por un microorganismo poco común. MÉD.UIS. 2017;30(3):121-7.

Palabras claves: Hepatitis Autoinmune. Bacteremia. Aeromonas. Autoanticuerpos. Autoinmunidad.

\section{Bacteremia caused by Aeromonas caviae in-patient with autoinmune hepatitis: case report}

\section{ABSTRACT}

Autoimmune hepatitis is an inflammatory hepatic disease mediated by an autoimmune mechanism. This disorder has a broad clinical spectrum and should be suspected in the presence of elevated aminotransferases and immunoglobulins in a patient with histopathology hepatitis. Diagnosis is made by ruling out other causes of hepatitis (viral, secondary to drugs) and confirmation with positive autoantibodies. Treatment is based on corticosteroid monotherapy or its association with other immunomodulators, obtaining good therapeutic response rates. Rate of hospital infection in patients with hepatic cirrhosis ranges from 32-34\%, with bacteremia being the fourth most frequent source, where Aeromonas represents $5 \%$ of these cases. We report the case of a 51-year-old patient with autoimmune hepatitis who consults for decompensation of secondary liver disease with a rare microorganism. MÉD.UIS. 2017;30(3):121-7.

Keywords: Aeromonas. Autoantibodies. Autoimmunity. Bacteremia. Hepatitis, Autoimmune.

¿Cómo citar este artículo?: Palmezano Díaz JM, Figueroa-Pineda CL, Rangel Rivera DA. Bacteremia por Aeromonas caviae en paciente con hepatitis autoinmune: Presentación de Caso. MÉD.UIS. 2017;30(3):121-7. 


\section{INTRODUCCIÓN}

La Hepatitis Autoinmune (HAI) es un trastorno inflamatorio progresivo hepático mediado por un proceso de autoinmunidad ${ }^{1,2}$. Esta patología presenta hallazgos inflamatorios en el estudio histopatológico asociados a aumento de aminotransferasas, autoanticuerpos orgánicos no específicos circulantes y elevación de inmunoglobulinas². Su epidemiología a nivel mundial es desconocida puesto que pocos trabajos se han realizado al respecto. Sin embargo, diversos estudios realizados en países como España, Dinamarca, Reino Unido, Suecia y Japón indican una prevalencia entre 11 y 24 casos por cada 100 000 habitantes $^{3-7}$. De igual forma, se calcula una incidencia que oscila entre $0,8-3,5$ casos nuevos por cada 100000 habitantes $^{8}$.

A pesar de no conocerse la causa específica de la enfermedad, se considera que se desarrolla en personas con predisposición genética que se exponen a algún agente desencadenante como bacterias, virus, hongos u otras partículas externas al organismo9,10; la caracterización del cuadro clínico es de amplio rango presentándose desde formas asintomáticas solo detectables por elevaciones moderadas de las aminotransferasas hasta casos de hepatitis severa fulminante ${ }^{1,11,12}$. De esta forma, la mayoría de los pacientes son asintomáticos o presentan formas leves de la enfermedad, siendo los síntomas generales inespecíficos como astenia, fatiga y debilidad un motivo de consulta frecuente. Otros pacientes consultan por ictericia, artralgias sin artritis o por complicaciones derivadas de la hipertensión portal tales como sangrado gastrointestinal o hiperesplenismo\%.

El diagnóstico de $\mathrm{HAl}$ se basa en la presencia de transaminasas elevadas, con aumento de inmunoglobulinas (especialmente $\lg G$ ), anticuerpos positivos y estudio histopatológico de hepatitis de interfase; dependiendo de la positividad del tipo de anticuerpos circulantes se clasifica en dos grupos: la tipo 1, para anticuerpos antinucleares (ANAS) y/o anticuerpos anti músculo liso; y la 2 con anticuerpos anticitosol hepático tipo 10 anticuerpo antimicrosomal de hígado/riñón tipo $1^{8}$. También, se pueden utilizar los sistemas de puntajes del International Autoimmune Hepatitis Group (Ver Tabla 1 y 4$)^{13,14}$, creados inicialmente para estandarizar el diagnóstico en estudios de investigación, siendo cada vez más utilizados en pacientes con clínica y hallazgos de laboratorios no típicos o escasos.
Tabla 1. Sistema de puntuación para el diagnóstico de hepatitis autoinmune

\begin{tabular}{|c|c|c|}
\hline Característica & Factor & Puntaje \\
\hline Sexo & Mujer & 2 \\
\hline \multirow{3}{*}{$\begin{array}{l}\text { Relación FA/AST (o ALT) } \\
\text { relación }\end{array}$} & $<1,5$ & 2 \\
\hline & $1,5-3,0$ & 0 \\
\hline & $>3,0$ & -2 \\
\hline \multirow{3}{*}{$\begin{array}{l}\text { Globulinas séricas o lgG } \\
\text { (veces límite superior de } \\
\text { referencia) }\end{array}$} & $1,5-2,0$ & 2 \\
\hline & $1,0-1,5$ & 1 \\
\hline & $<1,0$ & 0 \\
\hline \multirow{4}{*}{ ANA, SMA, anti-LKM-1 } & $>1: 80$ & 3 \\
\hline & 180 & 2 \\
\hline & $1: 40$ & 1 \\
\hline & $<1: 40$ & 0 \\
\hline AMA & Positivos & -4 \\
\hline \multirow{2}{*}{ Marcadores virales } & Positivos & -3 \\
\hline & Negativos & 3 \\
\hline \multirow{2}{*}{ Fármacos hepatotóxicos } & Sí & -4 \\
\hline & No & 2 \\
\hline \multirow{2}{*}{$\begin{array}{l}\text { Consumo medio de } \\
\text { alcohol }\end{array}$} & Menor 25 g/día & 2 \\
\hline & Mayor 60 g/día & -2 \\
\hline \multirow{6}{*}{$\begin{array}{l}\text { Características } \\
\text { histológicas }\end{array}$} & Hepatitis de interfase & 3 \\
\hline & Células plasmáticas & 1 \\
\hline & Rosetas & 1 \\
\hline & Nada de lo anterior & -5 \\
\hline & Cambios biliares* & -3 \\
\hline & Cambios atípicos** & -3 \\
\hline $\begin{array}{l}\text { Enfermedades } \\
\text { autoinmunes }\end{array}$ & Tiroiditis, colitis, otras & 2 \\
\hline HLA & DR3 o DR4 & 1 \\
\hline $\begin{array}{c}\text { Seropositivas para otros } \\
\text { anticuerpos }\end{array}$ & $\begin{array}{c}\text { Anti SLA/LP, actina, } \\
\text { pANCAS }\end{array}$ & 2 \\
\hline \multirow{2}{*}{ Respuesta al tratamiento } & Remisión & 2 \\
\hline & Remisión y recividad & 3 \\
\hline
\end{tabular}

Cortes pretratamiento: 10-15: probable HAI; mayor de 15: HAI definitiva

Cortes postratamiento: 12-17: probable HAl; mayor de 17: HAI definitiva.

FA: Fosfatasa Alcalina; AST: Aspartato Aminotransferasa; ALT: Alanino Aminotransferasa; IgG: Inmunoglobulina G;

ANA: Anticuerpos Antinucleares; SMA: Anticuerpos Contra Músculo Liso; anti-LKM-1: Anticuerpo Antimicrosomal de Hígado/ Riñón; AMA: Anticuerpo Antimitocondriales; HLA: Antígeno Leucocitario Humano; pANCAS: Anticuerpos Anticitoplasma de Neutrófilo de Patrón de Fluorescencia Perinuclear

* Incluyendo colangitis granulomatosa, fibrosis periductal concéntrica, ductopenia, proliferación biliar ductal marginal y colangiolitis.

**Otras características sugestivas de otra enfermedad.

Fuente: Adaptado de: Alvarez F, Berg PA, Bianchi FB, Bianchi L, Burroughs AK, Cancado EL, et al. International Autoimmune Hepatitis Group Report: review of criteria for diagnosis of autoimmune hepatitis. Journal of hepatology. 1999;31(5):929-38. ${ }^{13}$ 
El género Aeromonas agrupa una serie de bacterias gram negativas anaerobias facultativas localizados en el agua, carne y vegetales, con la capacidad de colonizar tanto al humano como a los animales ${ }^{15}$. Estas se relacionan principalmente con casos de gastroenteritis, bacteriemia e infecciones de la vía biliar. Otras formas de infección menos usuales han sido reportadas, e incluyen infecciones pulmonares, meningitis, peritonitis y síndrome hemolítico urémico $^{16}$. Este grupo de bacterias representa alrededor del 5\% de los casos de bacteriemia en pacientes con cirrosis hepática ${ }^{17}$ y una mortalidad entre el 30-50\% en sepsis ${ }^{16,18,19}$, siendo mayor y más severa en aquellos infectados con $A$. hydrophila $y$ A. veronii en comparación con $A$. caviae, donde se reporta una mortalidad entre el 5-17\% de los $\operatorname{casos}^{16,20}$.

En este artículo se presenta el caso de una paciente femenina adulta media ingresada por descompensación de $\mathrm{HAl}$ asociada a sepsis por Aeromonas Caviae, agente poco común como causa de bacteremia en nuestro medio. Se resalta la importancia del adecuado estudio de los pacientes con hepatopatía crónica de causa no clara para administrar tratamiento oportuno y mejorar su pronóstico.

\section{Presentación de caso clínico}

Paciente femenina de 51 años natural y procedente de zona urbana de Bucaramanga, Colombia quien consultó por cuadro clínico de ocho días de evolución de dolor abdominal generalizado irradiado a reja costal derecha con aumento progresivo de perímetro abdominal. De forma asociada, presentaba edema en miembros inferiores, disnea de medianos esfuerzos, astenia, adinamia e hiporexia.

Como antecedentes personales, paciente con diabetes mellitus tipo 2 e hipertensión arterial. De igual forma, había presentado episodio de hemorragia de vías digestivas altas en el año 2012 con estudio endoscópico que evidenció varices esofágicas grado III y micro úlceras duodenales Forrest IIc, recibiendo tratamiento con ligadura en tres ocasiones; en ese mismo año, recibe diagnóstico de cirrosis hepática sin etiología identificada. Como tratamiento para patologías antes mencionadas, recibía glibenclamida $5 \mathrm{mg}$ y propranolol $40 \mathrm{mg}$ cada 12 horas, enalapril $20 \mathrm{mg}$, espironolactona $25 \mathrm{mg}$ y omeprazol 20 mg cada día.

La paciente había sido valorada por servicio de ginecoobstetricia 6 días antes del ingreso por sangrado genital anormal indicando ácido tranexámico 500 mg cada día. Negaba infecciones de transmisión sexual, transfusión de hemoderivados, consumo de bebidas alcohólicas, tabaquismo y uso de drogas endovenosas. Refería administración de esquema de vacunación completo en la niñez, pero no portaba documentos que certificaran dicha información.

Al examen físico de ingreso se encontró signos vitales dentro de normalidad, conjuntivas ictéricas, abdomen distendido con onda ascítica positiva y dolor difuso a la palpación profunda; edema grado II de miembros inferiores con fóvea, sin varices. Resto de examen dentro de normalidad. Se solicitaron estudios iniciales (Ver Tabla 2). Además, se realizó paracentesis con líquido sin criterios para infección intraabdominal y con gradiente albúmina/albúmina de 2,3 sugestivo de hipertensión portal.

Es valorada por el servicio de gastroenterología considerando paciente sin antecedentes tóxicos o de infección por virus hepatotropos que cursaba con hepatopatía crónica descompensada sin etiología clara por lo que se indicó la realización de estudios complementarios de autoanticuerpos (Ver Tabla 3). Al cuarto día de evolución la paciente presentó deterioro clínico dado por picos febriles, hipotensión, hipoglicemia, acidosis metabólica, leucocitosis (22 000 $x \mathrm{~mm}^{3}$ ) e hiperlactatemia (7,1 $\left.\mathrm{mmol} / \mathrm{L}\right)$.

Por los hallazgos anteriores, se inició cubrimiento antibiótico de amplio espectro con vancomicina y piperacilina tazobactam asociado a soporte vasopresor (norepinefrina) como parte del protocolo de manejo de shock séptico. Se estudiaron posibles focos infecciosos con parcial de orina, cultivo de líquido peritoneal, radiografía de tórax y tomografía axial computarizada de tórax, todos estos estudios dentro de normalidad, con excepción de derrame pleural bilateral con atelectasias pasivas (Ver Figura 1). La paciente presentó buena evolución clínica, sin nuevos picos febriles en días posteriores, con tolerancia a destete de norepinefrina y disminución de lactato sérico.

Posteriormente, se recibieron hemocultivos positivos para Aeromonas Caviae multirresistente, realizando cambio de tratamiento guiado por antibiograma a meropenem, tratamiento que recibió durante 10 días con evolución satisfactoria, con nuevos hemocultivos negativos. Se reciben demás estudios de autoanticuerpos que orientan hacia el diagnóstico 
de HAl tipo 2 (Ver Tabla 4). Se consideró que los anteriores hallazgos en ausencia de otra causa que explicara la hepatopatía crónica, permitía realizar el diagnóstico clínico de HAI.

Tabla 2. Estudios de laboratorio solicitados al ingreso

\begin{tabular}{|c|c|}
\hline Estudio & Resultado \\
\hline Leucocitos & $7.150 \times \mathrm{mm}^{3}$ \\
\hline Hemoglobina & $13,2 \mathrm{~g} / \mathrm{dL}$ \\
\hline Hematocrito & $43,10 \%$ \\
\hline Plaquetas & $100000 \times \mathrm{mm}^{3}$ \\
\hline Neutrófilos & $57,40 \%$ \\
\hline Linfocitos & $29,80 \%$ \\
\hline Antígeno S hepatitis B & Negativo \\
\hline Anticuerpos hepatitis C & Negativo \\
\hline ELISA-VIH & Negativo \\
\hline BUN & $7,39 \mathrm{mg} / \mathrm{dL}$ \\
\hline Creatinina & $0,61 \mathrm{mg} / \mathrm{dL}$ \\
\hline Glicemia & $79 \mathrm{mg} / \mathrm{dl}$ \\
\hline Hemoglobina glicosilada & $5,10 \%$ \\
\hline Bilirrubina total & $6,3 \mathrm{mg} / \mathrm{dL}$ \\
\hline Bilirrubina directa & $4,47 \mathrm{mg} / \mathrm{dL}$ \\
\hline Aspartato aminotransferasa & $97,3 \mathrm{mg} / \mathrm{dL}$ \\
\hline Alanino aminotransferasa & $45,8 \mathrm{mg} / \mathrm{dL}$ \\
\hline Fosfatasa alcalina & $144,0 \mathrm{mg} / \mathrm{dL}$ \\
\hline Sodio & $137,0 \mathrm{mEq} / \mathrm{l}$ \\
\hline Potasio & $4,65 \mathrm{mEq} / \mathrm{l}$ \\
\hline Cloro & $103,2 \mathrm{mEq} / \mathrm{l}$ \\
\hline Calcio total & $8,5 \mathrm{mg} / \mathrm{dL}$ \\
\hline Proteínas totales & $6,63 \mathrm{~g} / \mathrm{dL}$ \\
\hline Albúmina & $2,74 \mathrm{~g} / \mathrm{dL}$ \\
\hline Tiempo de protrombina & 23,7 segundos \\
\hline INR & 1,88 \\
\hline Tiempo de tromboplastina & 44,5 segundos \\
\hline
\end{tabular}

Fuente: Autores.
Tabla 3. Estudio de autoanticuerpos solicitados al paciente

\begin{tabular}{|c|l|l|}
\hline Estudio & Resultado & Valor de referencia \\
\hline ANAS-2012 & $96,4 \mathrm{Uds}$. & Menor de 25,0 Uds. \\
\hline ANAS-2015 & $21,8 \mathrm{Uds}$. & Negativo: <20 uds \\
\hline Inmunoglobulina IgG & $\begin{array}{l}2368,4 \\
\mathrm{mg} / \mathrm{dl}\end{array}$ & $700-1600 \mathrm{mg} / \mathrm{dl}$ \\
\hline $\begin{array}{c}\text { Anticuerpos anti } \\
\text { músculo liso }\end{array}$ & 180 & Menor de 1:40 \\
\hline $\begin{array}{c}\text { Anticuerpos } \\
\text { antimicrosomales }\end{array}$ & $54,6 \mathrm{UI} / \mathrm{mL}$ & $\begin{array}{l}\text { Negativo: menor de } \\
25 \text { Ul/mL }\end{array}$ \\
\hline $\begin{array}{c}\text { AMA (Anticuerpos } \\
\text { antimitocondriales) }\end{array}$ & $22,8 \mathrm{U}$ & $\begin{array}{l}\text { Negativo: menor o } \\
\text { igual a 20 U }\end{array}$ \\
\hline $\begin{array}{c}\text { Anticuerpos } \\
\text { anticitoplasma de } \\
\text { neutrófilo (ANCAS)- } \\
\text { patrón pANCA }\end{array}$ & negativo & $\begin{array}{l}\text { Significativo: título } \\
\text { mayor o igual a } \\
1: 20 .\end{array}$ \\
\hline $\begin{array}{c}\text { Anticuerpos } \\
\text { anticitoplasma de } \\
\text { neutrófilo (ANCAS)- } \\
\text { patrón cANCA }\end{array}$ & $1: 20$ & $\begin{array}{l}\text { Significativo: título } \\
\text { mayor o igual a } \\
1: 20 .\end{array}$ \\
\hline
\end{tabular}

Fuente: Autores.

Se tomó una biopsia hepática antes del egreso hospitalario para realizar confirmación histopatológica con hepatitis crónica severa (Ver Figura 2 y 3). Dados los hallazgos anteriores junto con el resultado de autoanticuerpos se decidió iniciar tratamiento de ciclo intensivo por cuatro semanas con prednisona (la primera semana con $30 \mathrm{mg} /$ día; $20 \mathrm{mg} /$ día en la segunda semana; y $15 \mathrm{mg} /$ día por dos semanas siguientes), azatriopina (50 mg/día) y continuar manejo de cirrosis hepática por parte de servicio de medicina interna y gastroenterología al igual que valoración ambulatoria por el servicio de trasplantes. También se indicó vacunación para hepatitis A, hepatitis B y PPD.

\section{Discusión}

La HAl es una enfermedad heterogénea con edad de presentación variable que afecta a cualquier raza, y, de no ser tratada, puede progresar de forma frecuente a cirrosis $^{10}$. El sistema de puntaje inicial descrito en 1999 tiene la posibilidad de ser aplicado antes o después del tratamiento inmunosupresor (Ver Tabla 1) ${ }^{13}$. Al aplicarlo a nuestro paciente se obtuvo un valor mayor a 15, confirmando el diagnóstico. Además, se aplicó un sistema más corto y práctico que ha sido recomendado de forma reciente que también lo confirmó, con un puntaje de 7 (Ver Tabla 4) ${ }^{14}$. 


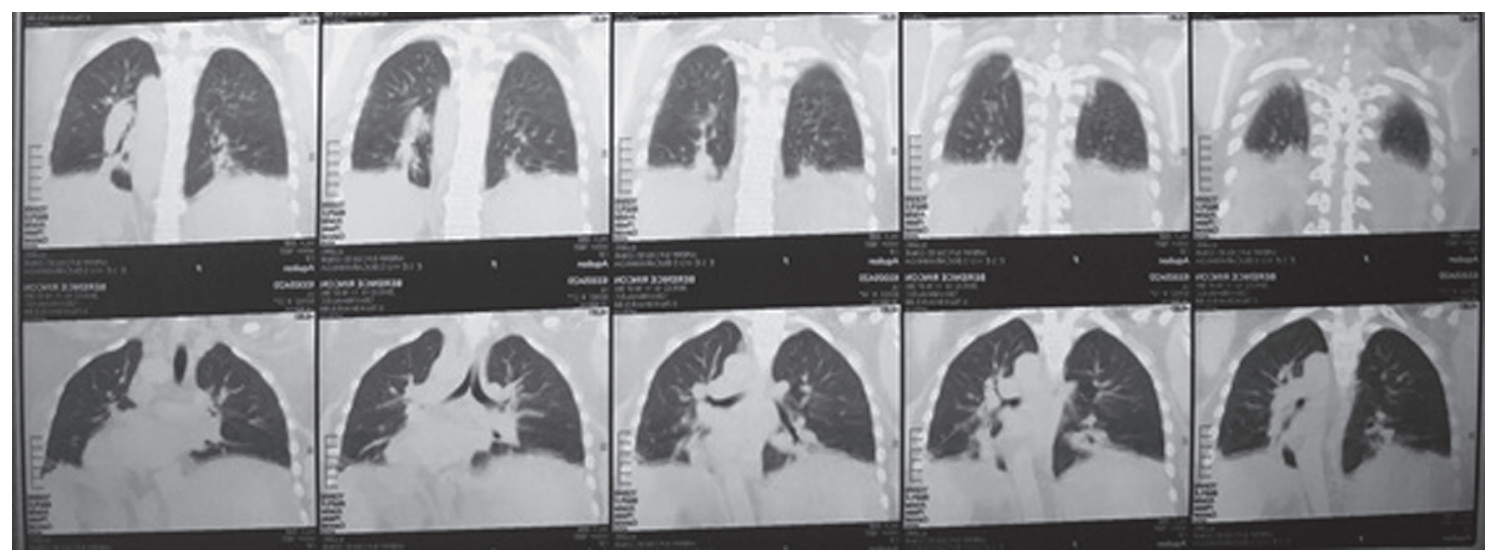

Figura 1. Tomografía axial computarizada de tórax

Derrame pleural bilateral de predominio derecho con atelectasias pasivas. Pequeña banda atelectásica en língula.

Fuente: Autores.

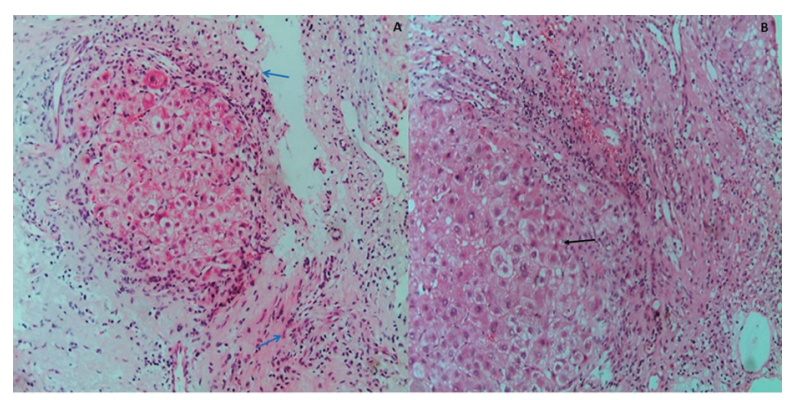

Figura 2. Biopsia Hepática

A. Abundante infiltrado mononuclear linfoplasmocitario (flecha azul). B. Degeneración balonizante de hepatocitos (flecha negra). (A y B, tinción HE; A: 40X; B: 40X).

Fuente: Departamento de Patología, Universidad Industrial de Santander.

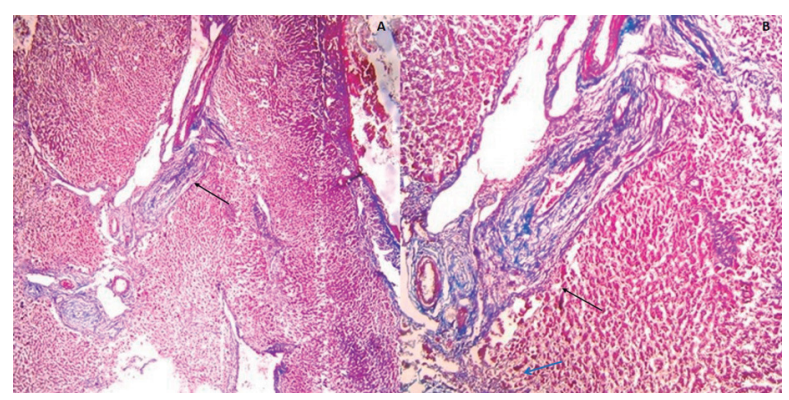

Figura 3. Biopsia Hepática

A, B. Abundante fibrosis porto portal y numerosos septos fibrosos con cirrosis (flecha negra). Parénquima hepático con evidencia de infiltrado inflamatorio de predominio mononuclear asociado a necrosis periportal (flecha azul). (A y $B$, tinción tricrómico de Masson; A: 4X; B: 10X).

Fuente: Departamento de Patología, Universidad Industrial de Santander.

En pacientes con cirrosis hepática hospitalizados se presentan tasas de infección que oscilan entre 32$34 \%{ }^{21}$. La fuente de estas infecciones son en orden de frecuencia: peritonitis espontánea, infección de vías urinarias, neumonía, bacteremia e infecciones de piel y tejidos blandos ${ }^{22}$. La mayoría de estas infecciones son causadas por Escherichia coli, Klebsiella spp., Enterobacter spp. y P. aeruginosa ${ }^{23}$. En el reporte presentado, el origen de la sepsis fue una bacteremia descartando previamente los otros focos más frecuentes.

En la bacteremia por el género Aeromonas es poco frecuente identificar la fuente de la infección, indicando como localización más probable la flora intestinal endógena ${ }^{24,25}$. En pacientes cirróticos, lo anterior se ha relacionado con la presencia de translocación bacteriana por alteración de la barrera intestinal producto del edema, congestión e hipoxia local ${ }^{26,27}$. En el caso presentado, no fue posible identificar heridas, infecciones en piel o trauma previo al inicio del cuadro clínico, por lo que se consideró como fuente más probable de transmisión la vía oral como se ha reportado en otros casos similares ${ }^{23,27}$.

Los estudios muestran que los pacientes con bacteremia por Aeromonas generalmente presentan inmunosupresión, neoplasias malignas o hepatopatías ${ }^{19,28}$. Éstas últimas se han vinculado con mayor susceptibilidad a desarrollar infecciones generalizadas por ciertos agentes infecciosos poco comunes en huéspedes inmunocompetentes debido a alteraciones en el sistema inmune. Dentro de las anteriores se destacan una producción alterada de opsoninas, producción deficiente de las proteínas del sistema de complemento y una función alterada de las células de Kupffer ${ }^{29}$.

La identificación precoz y el tratamiento de la sepsis y el shock séptico son primordiales para mejorar el pronóstico. Además de la terapia de sostenimiento, el régimen antibiótico recomendado para tratamiento 
de infección por Aeromonas incluye el uso de carbapenémicos o una asociación de cefalosporinas de tercera generación y aminoglucósidos o fluoroquinolonas ${ }^{21}$. En la paciente se utilizó un régimen basado en meropenem durante 10 días con buena respuesta terapéutica en las primeras 24 horas.

Tabla 4. Criterios simplificados para el diagnóstico de hepatitis autoinmune

\begin{tabular}{|c|c|c|c|}
\hline Característica & Valor & Puntaje & $\begin{array}{l}\text { Puntaje } \\
\text { paciente }\end{array}$ \\
\hline ANA o SMA & $\begin{array}{l}\text { Mayor o igual a } \\
1: 40\end{array}$ & 1 & 1 \\
\hline ANA o SMA & $\begin{array}{l}\text { Mayor o igual a } \\
1: 80\end{array}$ & \multirow{3}{*}{$2^{*}$} & \multirow{3}{*}{2} \\
\hline 0 anti-LKM-1 & $\begin{array}{l}\text { Mayor o igual a } \\
1: 40\end{array}$ & & \\
\hline O SLA & Positivo & & \\
\hline \multirow[b]{2}{*}{$\lg G$} & $\begin{array}{l}\text { Mayor al límite } \\
\text { normal }\end{array}$ & 1 & - \\
\hline & $\begin{array}{l}\text { Mayor a 1,10 } \\
\text { veces el límite } \\
\text { normal }\end{array}$ & 2 & 2 \\
\hline \multirow[t]{2}{*}{$\begin{array}{c}\text { Hallazgos } \\
\text { histopatológicos }\end{array}$} & $\begin{array}{l}\text { Compatible con } \\
\mathrm{HAl}\end{array}$ & 1 & 1 \\
\hline & $\begin{array}{l}\text { Hallazgos } \\
\text { típicos de HAl }\end{array}$ & 2 & -- \\
\hline $\begin{array}{c}\text { Ausencia de } \\
\text { hepatitis viral }\end{array}$ & Si & 2 & 2 \\
\hline
\end{tabular}

Puntajes de corte: mayor o igual a 6: probable HAl; mayor o igual a 7: diagnóstico de $\mathrm{HAl}$.

ANA: Anticuerpos Antinucleares; SMA: Anticuerpos Contra Músculo Liso; anti-LKM-1: Anticuerpo Antimicrosomal de Hígado/Riñón Tipo 1; SLA: Anticuerpos contra el Antígeno Soluble Hepático; IgG: inmunoglobulina G; HAl: Hepatitis Autoinmune.

*Puntos totales para la variable anticuerpos no puede ser superior a 2. Fuente: Adaptado de: Hennes EM, Zeniya M, Czaja AJ, Pares A, Dalekos GN, Krawitt EL, et al. Simplified criteria for the diagnosis of autoimmune hepatitis. Hepatology. 2008;48(1):169-76. ${ }^{14}$

En cuanto al tratamiento de la $\mathrm{HAl}$ requiere la utilización de medicamentos inmunosupresores con el objetivo de mejorar sintomatología, reducir y revertir la fibrosis hepática y mejorar la supervivencia. Los esquemas de tratamiento más recomendados corresponden a prednisona o prednisolona en combinación con azatriopina dado los buenos resultados que sustentan su uso ${ }^{1,30}$. Se ha documentado la mejoría de los síntomas, la función hepática y la actividad inflamatoria hepática en aproximadamente el $80 \%$ de los pacientes con $\mathrm{HAl}$ que reciben corticoesteroides ${ }^{31}$. De igual forma, la supervivencia a 5 años aumenta a más del doble, alcanzando valores cercanos al 90\%32.

A pesar de que la $\mathrm{HAl}$ tiene baja prevalencia, se debe tener en cuenta como una causa de cirrosis y disfunción hepática de origen no claro. Si bien este trabajo no tiene peso estadístico, puede ser utilizado para realizar otras investigaciones y como parte de la educación médica de manera que se optimice el estudio de estos pacientes y se realice un manejo adecuado.

\section{CONCLUSIONES}

Las infecciones bacterianas en pacientes con cirrosis hepática son un problema con alta morbimortalidad. Por lo anterior, todos los pacientes con esta condición clínica deberían ser educados para acudir de forma inmediata a un centro médico cuando presenten fiebre o se sientan enfermos, y de esta manera realizar un estudio completo orientado a la identificación de un foco infeccioso primario con observación médica que permita realizar un tratamiento multidisciplinario e inicio oportuno de terapia antibiótica en caso de ser necesario.

La mayoría de las infecciones en pacientes con cirrosis se producen por bacterias gram negativas, pero otros tipos de microorganismos menos comunes siempre deben tenerse en cuenta. Se recomienda realizar siempre un estudio etiológico en los pacientes con cirrosis hepática de origen no claro. De esta manera, se podría brindar tratamiento médico específico con mejoría del pronóstico y la calidad de vida del paciente como en los casos de hepatitis autoinmune.

\section{COMItÉ DE ÉTICA}

Se contó con consentimiento informado por parte de la paciente para la publicación de la información, incluyendo el resultado del estudio histopatológico y las imágenes diagnósticas presentadas.

\section{FinANCIAMIENTO}

No se recibió patrocinio de ningún tipo para llevar a cabo este artículo.

\section{CONFLICTO DE INTERESES}

Los autores declaran no tener ningún conflicto de intereses. 


\section{Referencias Biblográficas}

1. Czaja AJ. Diagnosis and management of autoimmune hepatitis. Clin Liver Dis. 2015;19(1):57-79.

2. Manns MP, Lohse AW, Vergani D. Autoimmune hepatitisUpdate 2015. J Hepatol. 2015;62Suppl:s100-11.

3. Werner M, Prytz H, Ohlsson B, Almer S, Bjornsson E, Bergquist A, et al. Epidemiology and the initial presentation of autoimmune hepatitis in Sweden: a nationwide study. Scand J Gastroenterol. 2008;43(10):1232-40

4. Boberg KM, Aadland E, Jahnsen J, Raknerud N, Stiris M, Bell H. Incidence and prevalence of primary biliary cirrhosis, primary sclerosing cholangitis, and autoimmune hepatitis in a Norwegian population. Scand J Gastroenterol. 1998;33(1):99-103.

5. Primo J, Maroto N, Martinez M, Anton MD, Zaragoza A, Giner $\mathrm{R}$, et al. Incidence of adult form of autoimmune hepatitis in Valencia (Spain).Acta Gastroenterol Belg. 2009;72(4):402-6.

6. Whalley S, Puvanachandra P, Desai A, Kennedy H. Hepatology outpatient service provision in secondary care: a study of liver disease incidence and resource costs. J R Coll Physicians Lond. 2007;7(2):119-24.

7. Abe M, Mashiba T, Zeniya M, Yamamoto K, Onji M, Tsubouchi $\mathrm{H}$, et al. Present status of autoimmune hepatitis in Japan: a nationwide survey. J Gastroenterol. 2011:46(9):1136-41.

8. Liberal R, Vergani D, Mieli-Vergani G. Update on Autoimmune Hepatitis. J Clin Transl Hepatol. 2015;3(1):42-52.

9. Gatselis NK, Zachou K, Koukoulis GK, Dalekos GN. Autoimmune hepatitis, one disease with many faces: etiopathogenetic, clinicolaboratory and histological characteristics. World J Gastroenterol. 2015;21(1):60-83.

10. Moy L, Levine J. Autoimmune hepatitis: a classic autoimmune liver disease. Curr Probl Pediatr Adolesc Health Care. 2014;44(11):341-6

11. Kogan J, Safadi R, Ashur Y, Shouval D, Ilan Y. Prognosis of symptomatic versus asymptomatic autoimmune hepatitis: a study of 68 patients. J Clin Gastroenterol. 2002;35(1):75-81.

12. Kessler WR, Cummings OW, Eckert G, Chalasani N, Lumeng L, Kwo PY. Fulminant hepatic failure as the initial presentation of acute autoimmune hepatitis. Clin Gastroenterol Hepatol. 2004;2(7):625-31.

13. Alvarez F, Berg PA, Bianchi FB, Bianchi L, Burroughs AK, Cancado EL, et al. International Autoimmune Hepatitis Group Report: review of criteria for diagnosis of autoimmune hepatitis. J Hepatol. 1999;31(5):929-38.

14. Hennes EM, Zeniya M, Czaja AJ, Parés A, Dalekos GN, Krawitt EL, et al. Simplified criteria for the diagnosis of autoimmune hepatitis. Hepatology. 2008;48(1):169-76.

15. Khor WC, Puah SM, Tan JA, Puthucheary SD, Chua KH. Phenotypic and Genetic Diversity of Aeromonas Species Isolated from Fresh Water Lakes in Malaysia. PLoS ONE. 2015;10(12).

16. Chuang HC, Ho YH, Lay CJ, Wang LS, Tsai YS, Tsai CC. Different clinical characteristics among Aeromonas hydrophila, Aeromonas veronii biovar sobria and Aeromonas caviae monomicrobial bacteremia. J Korean Med Sci. 2011;26(11):1415-20.

17. Shizuma T, Tanaka C, Mori H, Fukuyama N. Investigation of Bacteremia due to Aeromonas Species and Comparison with That due to Enterobacteria in Patients with Liver Cirrhosis. Gastroenterol Res Pract. 2011;2011:1-4.

18. Janda JM, Guthertz LS, Kokka RP, Shimada T. Aeromonas species in septicemia: laboratory characteristics and clinical observations. Clin Infect Dis. 1994:19(1):77-83.

19. Ko WC, Lee HC, Chuang YC, Liu CC, Wu JJ. Clinical features and therapeutic implications of 104 episodes of monomicrobial Aeromonas bacteraemia. J Infect. 2000;40(3):267-73.

20. Ko WC, Chuang YC. Aeromonas bacteremia: review of 59 episodes. Clin Infect Dis. 1995;20(5):1298-304.

21. Bunchorntavakul C, Chavalitdhamrong D. Bacterial infections other than spontaneous bacterial peritonitis in cirrhosis. World J Hepatol. 2012;4(5):158-68.

22. Fernandez J, Navasa M, Gomez J, Colmenero J, Vila J, Arroyo V, et al. Bacterial infections in cirrhosis: epidemiological changes with invasive procedures and norfloxacin prophylaxis. Hepatology. 2002;35(1):140-8.

23. Yumoto T, Ichiba S, Umei N, Morisada S, Tsukahara K, Sato K, et al. Septic shock due to Aeromonas hydrophila bacteremia in a patient with alcoholic liver cirrhosis: a case report. J Med Case Rep. 2014; 8: 402.

24. Spadaro S, Berselli A, Marangoni E, Romanello A, Colamussi MV, Ragazzi R, et al. Aeromonas sobria necrotizing fasciitis and sepsis in an immunocompromised patient: a case report and review of the literature. J Med Case Rep. 2014; $8: 315$

25. Mandell GL, Bennett JE, Dolin R. Mandell, Douglas, and Bennett's principles and practice of infectious diseases. 7th ed. Philadelphia, PA: Churchill Livingstone/Elsevier; 2010.

26. Chiva M, Guarner C, Peralta C, Llovet T, Gomez G, Soriano G, et al. Intestinal mucosal oxidative damage and bacterial translocation in cirrhotic rats. Eur J Gastroenterol Hepatol. 2003;15(2):145-50.

27. Shin GW, You MJ, Cho HS, Yi SW, Lee CS. Severe sepsis due to Aeromonas aquariorum in a patient with liver cirrhosis. Jpn J Infect Dis. 2013; 66(6): 519-22.

28. Tsai MS, Kuo CY, Wang MC, Wu HC, Chien CC, Liu JW. Clinical features and risk factors for mortality in Aeromonas bacteremic adults with hematologic malignancies. J Microbiol Immunol Infect. 2006;39(2):150-4

29. Bogdanos DP, Gao B, Gershwin ME. Liver immunology. Compr Physiol. 2013;3(2):567-98

30. Gleeson D, Heneghan MA; British Society of G. British Society of Gastroenterology (BSG) guidelines for management of autoimmune hepatitis. Gut. 2011;60(12):1611-29.

31. Soloway RD, Summerskill WH, Baggenstoss AH, Geall MG, Gitnick GL, Elveback IR, et al. Clinical, biochemical, and histological remission of severe chronic active liver disease: a controlled study of treatments and early prognosis. Gastroenterology. 1972;63(5):820-33.

32. Delgado JS, Vodonos A, Malnick S, Kriger O, Wilkof-Segev R, Delgado B, et al. Autoimmune hepatitis in southern Israel: a 15year multicenter study. J Dig Dis. 2013;14(11):611-8. 